\title{
Research and implementation of remote monitoring system based on Internet of things
}

\author{
Zhencai $\mathrm{Li}^{1, a^{*}}$, Yawen Dai ${ }^{2, b^{*}}$ andJiawei Shen ${ }^{3, c}$ \\ ${ }^{1}$ Wuhan University of Technology, China \\ a627787952@qq.com, bdaiyaweny@163.com, c1069870257@qq.com
}

Keywords: Internet of things; remote monitoring; sensor; LabVIEW

\begin{abstract}
Commissioned by an aquaculture company, a remote monitoring system based on Internet of things technology was studied. It takes the Mega2560 processor as the core, the WiFi wireless RF data transmission as a means, the application of computer management, display software and networking platform will be the whole system to constitute a network of temperature and humidity monitoring and on-line real-time display, remote control. At the same time, the basic working principle of the system is introduced, the hardware structure of the system is described, and the main points of the design are analyzed, and the characteristics of the system are summarized. It is a typical application of the Internet of things technology in practical application.
\end{abstract}

\section{Introduction}

At present, the existing equipment monitoring system is based on the wired mode, and through the nodes, the relay equipment and the monitoring center, CAN, RS485 and other control network, the device node is incorporated into the control management system [1]. This can improve the monitoring efficiency, save the human cost, but the biggest drawback is the wired way is the wiring process is complex, the maintenance cost is high.

The Internet of things technology is a kind of network technology, which is based on RFID, infrared sensor, global positioning system, laser scanner, etc., According to the agreement, any goods and Internet connection, information exchange and communication to achieve intelligent identification, positioning, tracking, monitoring and management of a network technology. Because of its "physical and related" characteristics, the Internet of things has been widely used in the world, the typical logistics, monitoring, smart home, transportation and other industries.

This paper start from the basic concept of Internet of things, through the application of WiFi module, designed a remote monitoring system used in aquaculture monitoring. The system has simple structure, diverse functions, high cost performance, and is a case study of the successful application of the Internet of things.

\section{Design of system hardware}

The whole hardware structure of the system can be divided into four parts, data acquisition module, central control module, network module and computer. The data acquisition module is composed of sensor array, which is responsible for collecting temperature, humidity, oxygen content in water, and the central control module, which is responsible for processing the data collected by the data acquisition module. The network module is responsible for the data processing of MCU [2]. 


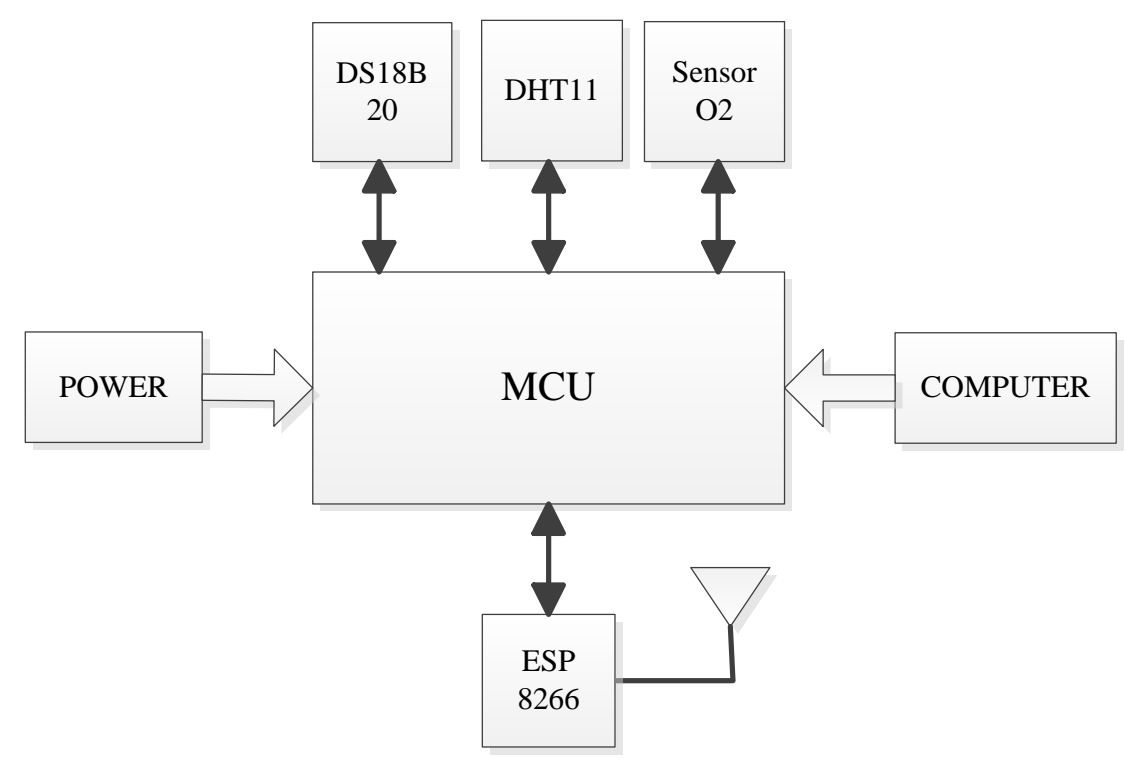

Fig.1. The whole hardware structure of the system

\section{A. The data acquisition module}

The main function of the data acquisition module is to select the appropriate sensor, the required temperature, humidity and oxygen content in the water, such as the amount of data to be transferred into the telecommunications number and transmission to MCU.

DS18B20 module is used as temperature sensor. DS18B20 is a commonly used temperature sensor with small size, low cost, strong anti-interference ability and high accuracy. Its working power supply: 3.0 5.5V/DC (which can be used for data line); DS18B2 uses a unique single line interface, in connection with the microprocessor only need a single line to achieve two-way communication between the microprocessor and DS18B20, the temperature range of $55 \sim+125$, the inherent temperature error 1 . The programmable resolution is 9 to 12 bits, the corresponding resolution temperature is $0.5,0.25,0.125$ and 0.0625 , respectively, which can achieve high precision temperature measurement [3].

DHT11 module is used as humidity sensor. DHT11 is a temperature and humidity sensor with calibrated digital signal output. The accuracy of $5 \% \mathrm{RH}$ is 2 , the humidity range is $20-90 \% \mathrm{RH}$, the temperature range is $0 \sim 50$. The author uses only its humidity data, which will be used as humidity sensor only [4].

The AZ8403 dissolved oxygen meter is used as oxygen sensor, which can detect 0 19.99mg/L oxygen content, resolution of $0.01 \mathrm{mg} / \mathrm{L}$, using RS232 communication protocol and microprocessor communication.

\section{B. The central control module}

The central control module is the processor, which is responsible for processing the data collected by the data acquisition module and connected with the computer through the WiFi module. This module mainly consists of Mega2560. Mega2560 Arduino is the core of the USB interface circuit board, with 54 digital input output, suitable for a large number of IO interface design. The core of the processor is ATmega2560, which has 54 digital input / output ports (16 of which can be used as the PWM output), the 16 analog input, 4 UART interface, a 16MHz crystal oscillator. Mega2560 Arduino can be powered by 3 ways, and can automatically select the power supply mode [5]. 


\section{The network module}

Network module uses ESP8266, ESP8266 is a complete and self-contained WiFi network solutions, which can run independently, also can be used as slave with other Host running. The author uses the wireless Internet connection to undertake the task of the WiFi adapter, and adds it to the design of micro controller, the connection is simple and easy to be connected with the SPI/SDIO interface. ESP8266 is high chip integration module, including antenna switch balun, power management converter, so only a very few external circuit, and including the front-end module, including the entire solution in the design of the PCB space to a minimum.

\section{The design of system software}

The system software is mainly divided into two parts: the central control module software and computer software. The central control module software is written in C language, and the computer management monitoring interface is used by LabVIEW software.

\section{A. The central control module program}

The center module is the process and control unit, which is responsible for processing the data collected by the sensor module and controlling the communication between the ESP8266 and the host computer. The software design includes single line interface and RS232, UART communication. First, the command is sent out by the software. Then the central control module sends the command to the field data acquisition module, and waits for the data feedback and collection node to transmit the data to the center control module. The paper also use a LCD to display the data.

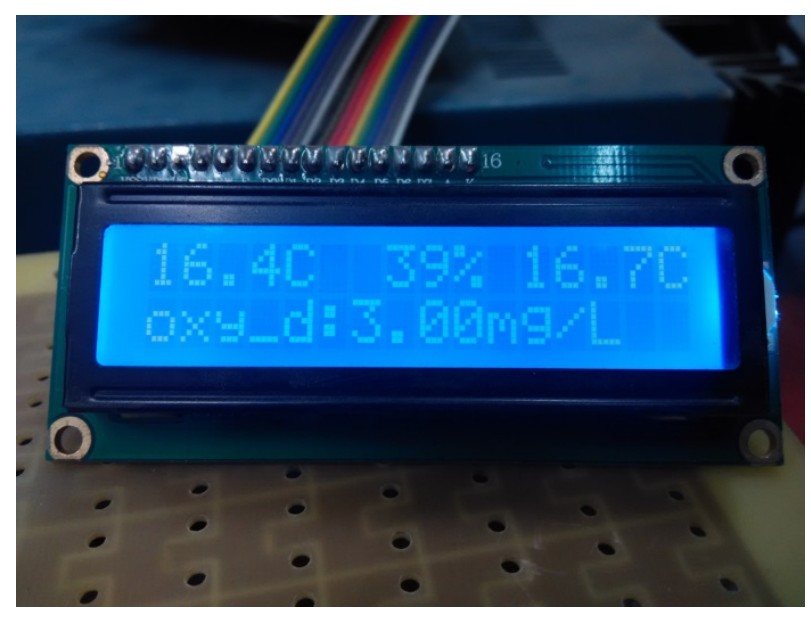

Fig.2. The data display

\section{B. The computer management monitoring interface}

LabVIEW is used as Computer software. It is developed by the national instrument (NI) company, similar to the $\mathrm{C}$ and BASIC development environment, but the LabVIEW and other computer language is a significant difference: other computer languages are used to generate code based on the text of the LabVIEW use of graphical editing language $G$ programming, the program is the form of block diagram. LabVIEW software is the core of the NI design platform, but also the ideal choice for developing measurement and control system [6].LabVIEW development environment integrates engineers and scientists to quickly build all the tools needed for various applications, designed to 
help engineers and scientists to solve problems, improve productivity and innovation.

The computer communication with the central control module for real-time communication using the TCP/IP, accessing to the IP 192.168.1.120 to carry out data exchange, the air humidity, temperature, air pressure, water content, water temperature and other data in the terminal equipment, and can be set up on the terminal platform switch operation of the remote device to avoid unnecessary labor costs.

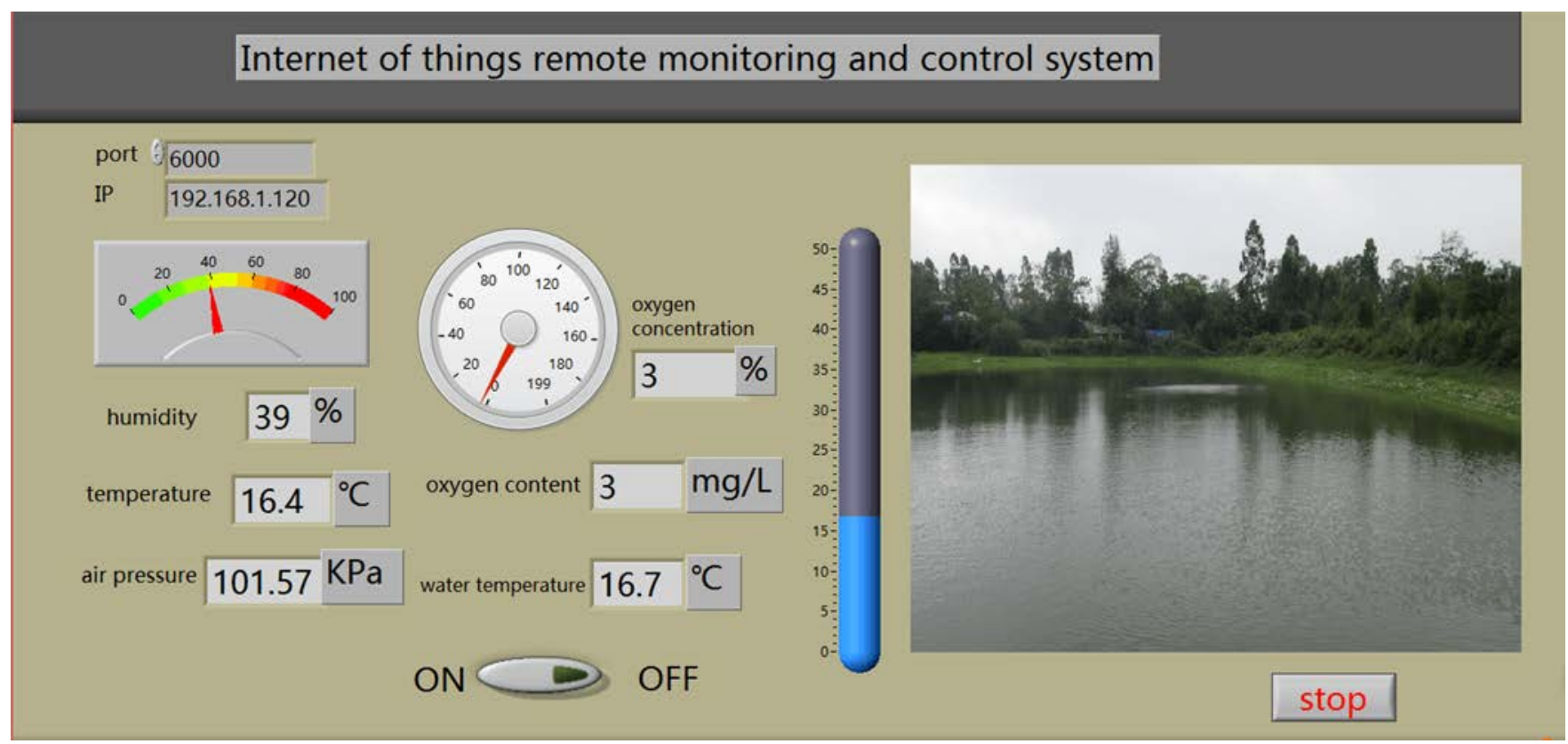

Fig.3. The computer management monitoring interface

\section{Conclusion}

This paper made a comprehensive test of the whole system, to achieve the desired performance, including temperature and humidity, dissolved oxygen sensor, wireless transmitting and receiving, computer management software, can work stably and reliably, and achieve the expected requirements of the system. In view of the current situation of automatic monitoring system which is lack of effective automatic monitoring system, this research is based on the idea of the Internet of things. The design of this system is simple and the function is perfect. The core technology of the system can be applied to the related industry, the market prospect is worth to be expected.

\section{References}

[1] Xing Xiaojiang, Wang Jianli, Li Mingdong. Services and Key Technologies of the Internet of Things. ZTE COMMUNICATIONS,June 2010 Vol.8 No.2.

[2] Li Xiaowei. Wireless Sensor Networks [M]. Beijing:Beijing Institute of Technology Press, 2007.

[3] https://www.maximintegrated.com/cn/products/analog/sensors-and-sensor-interface/DS18B20

[4] http://www.ic37.com/icpdf_datasheet_5/DHT11_datasheet_4458735/

[5] http://kb.open.eefocus.com/index.php?title=Arduino_Mega2560

[6] Sriharsha Vathsavayi, Hadaytullah, Kai Koskimies. Interleaving human and search-based software architecture design[J]. Proceedings of the Estonian Academy of Sciences, 2013, 62(1). 